Abstract We propose and demonstrate a new form of interferometry combining high phase sensitivity and excellent mechanical isolation. These features arise from maintaining a probe and reference tone in the same medium under test at all times, their ultimate homodyne detection, as well as a novel method employing an optical programmable filter which is used to extract their relative phase difference. We present a compilation of studies performed to illustrate the applicability of the scheme to a range of measurements of optical propagation characteristics. Measurements include the chromatic dispersion of SMF-28 of lengths ranging from $1 \mathrm{~m}$ to $1 \mathrm{~km}$, the nonlinear coefficient of highly nonlinear fibre and the phase transfer profile of an integrated optical ring resonator.

\title{
Spectral Difference Interferometry for the Characterisation of Optical Media
}

\author{
K. R. H. Bottrill ${ }^{1, *}$, M. A. Ettabib ${ }^{1}$, I. Demirtzioglou ${ }^{1}$, R. Marchetti ${ }^{2}$, C. Lacava ${ }^{1}$, F. Parmigiani ${ }^{3}$, \\ D.J. Richardson ${ }^{1}$ and P. Petropoulos ${ }^{1}$
}

\section{Introduction}

Inteferometry in general revolves around the interference of a probe tone which has been subject to some sort of phase shift due to propagation through the device-under-test (DUT), with a reference tone which has not. Most interferometers (such as the case with the well-known MachZehnder, Michelson and Sagnac interferometers [1]) split the probe and reference tones into two spatially separated modes over which they propagate before recombination and infer the relative phase difference between the two tones from the output power resulting from their coherent superposition. In the former two examples, the light is separated into two distinct spatial paths, whilst in the latter example, light is split into two directions of the same path which, although spatially overlapping, still constitute separate spatial modes.

In our proposed scheme, we take a different approach. Instead of separating the signal into two spatial modes, we separate it into two spectral modes using an electro-optic modulator. We then propagate these two tones, which could be considered to be the probe and reference tone, through the exact same optical path - the DUT, wherein they acquire a differential phase delay. In a traditional interferometer, the two tones must be returned to the same spatial mode to interfere and analogously, our tones too must be brought to the same frequency mode for the same reason. In our implementation, this is achieved using a second electrooptic modulator driven at the same frequency as the first.

One major challenge presented by multi-path schemes, such as the Mach-Zehnder and Michelson interferometer, is the maintenance of mutual coherency between the probe and reference tone until they interfere. This is usually achieved by maintaining the paths of both the probe and reference arms at similar lengths and isolating the system from mechanical vibrations to the greatest extent possible. The former places practical limitations upon the maximum length of device which can be measured using such schemes [2], whilst the latter complicates implementation and presents challenges in harsh environments. Sagnac-interferometers largely bypass such coherency challenges; provided that the transit time of the light is short compared to the time period of mechanical vibrations to which the interferometer is subject, light travelling in both directions will essentially pass through the medium in the same state and hence see the same optical path length. This accounts for the great stability of Sagnac interferometers, but propagation of the probe and signal tone at the same frequency through the same medium essentially precludes the direct application of such schemes to the measurement of frequency dependent phenomena such as chromatic dispersion. In our proposed scheme, as both the probe and signal tone copropagate in the same medium, in the same direction, the scheme is isolated from mechanical vibration in much the same way as the Sagnac interferometer, but without the same restriction on optical transit time, all the while being applicable to the measurement of phenomena such as chromatic dispersion.

A number of applications of the proposed technique are immediately apparent and we shall demonstrate three of these in this work: the first two, the measurement of chromatic dispersion and the determination of the Kerr nonlinear coefficient, are relevant to almost all optical media whilst the third, the phase transfer function of an integrated ring resonator, demonstrates the application of our scheme to the characterisation of photonic circuits, many of which are designed to have very specific phase and intensity characteristics. Beyond this, we envisage numerous future applications in sensing and characterisation.

\section{Concept}

For simplicity, we shall explain the operating principle of our scheme simultaneously with our implementation of it,

\footnotetext{
${ }^{1}$ University of Southampton $\quad{ }^{2}$ University of Pavia ${ }^{3}$ currently at Microsoft Research UK

* Corresponding author: e-mail: *krhb1g12@soton.ac.uk
} 
although it is important to note that other implementations are of course possible. Figure 1 provides a schematic of the experimental set-up used, along with spectra at notable stages of the lightpath. We separate the system into three stages, as shown in Figure 1, Probe Generation (orange panel), Probe Propagation (green panel) and finally Probe Detection (blue panel).

\subsection{Probe Generation}

The DUT is interrogated by a probe signal which consists of two coherent CW optical tones centred at $f_{c}$ with controllable relative phase and frequency separation $f_{s}$. To obtain these tones, a continuous wave (CW) laser (spectrum shown in Figure 1-1) is passed through a Mach-Zehnder modulator which is driven by an electrical sinusoid to create a simple coherent frequency comb of frequency spacing $f_{m}$ (Figure 1-2) [3]. The frequency comb is passed through a programmable optical filter (a Finisar Waveshaper 4000S), which is capable of applying a frequency dependent complex filter profile across the C-band. Using this filter, we suppress all but two lines with spacing $f_{s}=2 n f_{m}$ (where $n$ is an integer) constituting the two probe tones and apply a phase shift $\sigma$ to one of them (Figure 1-3), the role of which shall become clear later. Note, that whilst it is possible to select any two lines, we choose lines symmetric about the original carrier to simplify the following discussion. The resultant field is:

$$
\psi_{C}(t)=\psi_{+}(t)+\psi_{-}(t)=A_{+} e^{i\left[\omega_{+} t+\phi_{+}\right]}+A_{-} e^{i\left[\omega_{-} t+\phi_{-}+\sigma\right]}
$$

where the subscripts + and - indicate properties of the redand blue-shifted tones respectively and $f_{+}=f_{c}-f_{S} / 2$ and $f_{-}=f_{c}+f_{S} / 2$. Just before the two tones are launched into the DUT, it can be seen that the tones will already have each acquired a phase shift relative to the original carrier, not only due to the action of the comb, but also any other dispersive components in the lightpath up to the point of launch. To maintain generality we simply encapsulate the phase shifts experienced by the the red- and blue-shifted tones as $\phi_{+}$and $\phi_{-}$, respectively, and intentionally remain agnostic to their specific origin.

\subsection{Probe Propagation}

With the two probe tones generated, they may now be propagated through the DUT wherein they incur a phase shift, $\theta\left(\omega_{+}\right)$and $\theta\left(\omega_{-}\right)$, due to the propagation constant of the DUT of length, $L$, resulting in the following field:

$$
\psi_{C}(t)=B_{+} e^{i\left[\omega_{+} t+\phi_{+}+\theta\left(\omega_{+}\right) L\right]}+B_{-} e^{i\left[\omega_{-} t+\phi_{-}+\theta\left(\omega_{-}\right) L+\sigma\right]}
$$

where the magnitudes $A_{x}$ have been replaced by $B_{x}$ to indicate the effects of attenuation in the DUT. The properties of the medium we wish to measure are encoded in this phase shift. Figure 1-4 provides the spectrum of the tones after propagation. As expected, although the effects of attenuation can be seen in the spectrum, the phase shift induced by the DUT cannot be discerned (comparing Figure 1-3 and Figure 1-4), hence the need for interferometric techniques.

\subsection{Probe Detection}

To measure the phase difference, we pass the two tones through a second Mach-Zehnder modulator driven by the same electrical sinusoid as the first. This second modulator results in the creation of a comb from any $\mathrm{CW}$ tone entering it, but this time, since the input to the modulator is not one but two tones with frequency spacing $f_{S}=2 n f_{m}$, the result will be a series of overlapping side bands from each of the original tones (Figure 1-5). To illustrate this, Figure 2 shows the resultant combs produced by the second Mach-Zehnder modulator, a) - when only the blue-shifted tone enters, b) - when only the red-shifted tone enters and c) - when both tones enter. The tones from a) and b) which interfere to form c) are clearly visible in this figure. We focus our attention on the central tone formed at $f_{c}$ (highlighted in yellow in Figure 2), which we select using a second programmable filter. The power of the extracted tone is detected using a high sensitivity, slow photodetector connected to a transimpedence amplifier. We assume that the action of the second Mach-Zehnder modulator is to produce two tones at $f_{c}$, one arising from the tone at frequency $f_{+}$as well as a similar tone arising from the tone of frequency $f_{-}$:

$$
\begin{gathered}
\psi_{+}^{\prime}=B_{+}^{\prime} e^{i\left[\omega_{c} t+\phi_{+}^{\prime}(f)+\theta\left(\omega_{+}\right) L\right]} \\
\psi_{-}^{\prime}=B_{-}^{\prime} e^{i\left[\omega_{c} t+\phi_{-}^{\prime}\left(f_{c}\right)+\theta\left(\omega_{-}\right) L\right]}
\end{gathered}
$$

The presence of the primes in the magnitudes $B_{+}^{\prime}$ and $B_{-}^{\prime}$ indicate the potentially non-symmetric and non-unity conversion efficiency of the comb, whilst the primes in the phases $\phi_{+}^{\prime}$ and $\phi_{-}^{\prime}$ incorporate any relative phase shifts accrued by the two tones due to the action of the two combs as well as any dispersive component parts of the experimental set-up. As these two overlapping tones are coherent, not only because of their generation from the same original $\mathrm{CW}$ tone, but importantly also because of their copropagation throughout the set-up, the resultant field is of constant phase and power, effectively constituting a form of homodyne detection of the tone at $f_{c}$. At this stage, it would be possible to sweep the frequency of the original carrier $f_{c}$ and measure the power of this tone as it varies with $f_{c}$, effectively obtaining an interferogram from which the relative phase difference between the two tones could be inferred. In our approach, we instead exploit the phase shifting functionality of the programmable optical filter to extract the relative phase difference between the two tones unambiguously. By applying the phase shift $\sigma=\{0, \pi / 2, \pi, 3 \pi / 2\}$ radians to one of the tones and measuring the power of the tone produced by the inteference at $f_{c}$, we obtain the 
Probe Generation

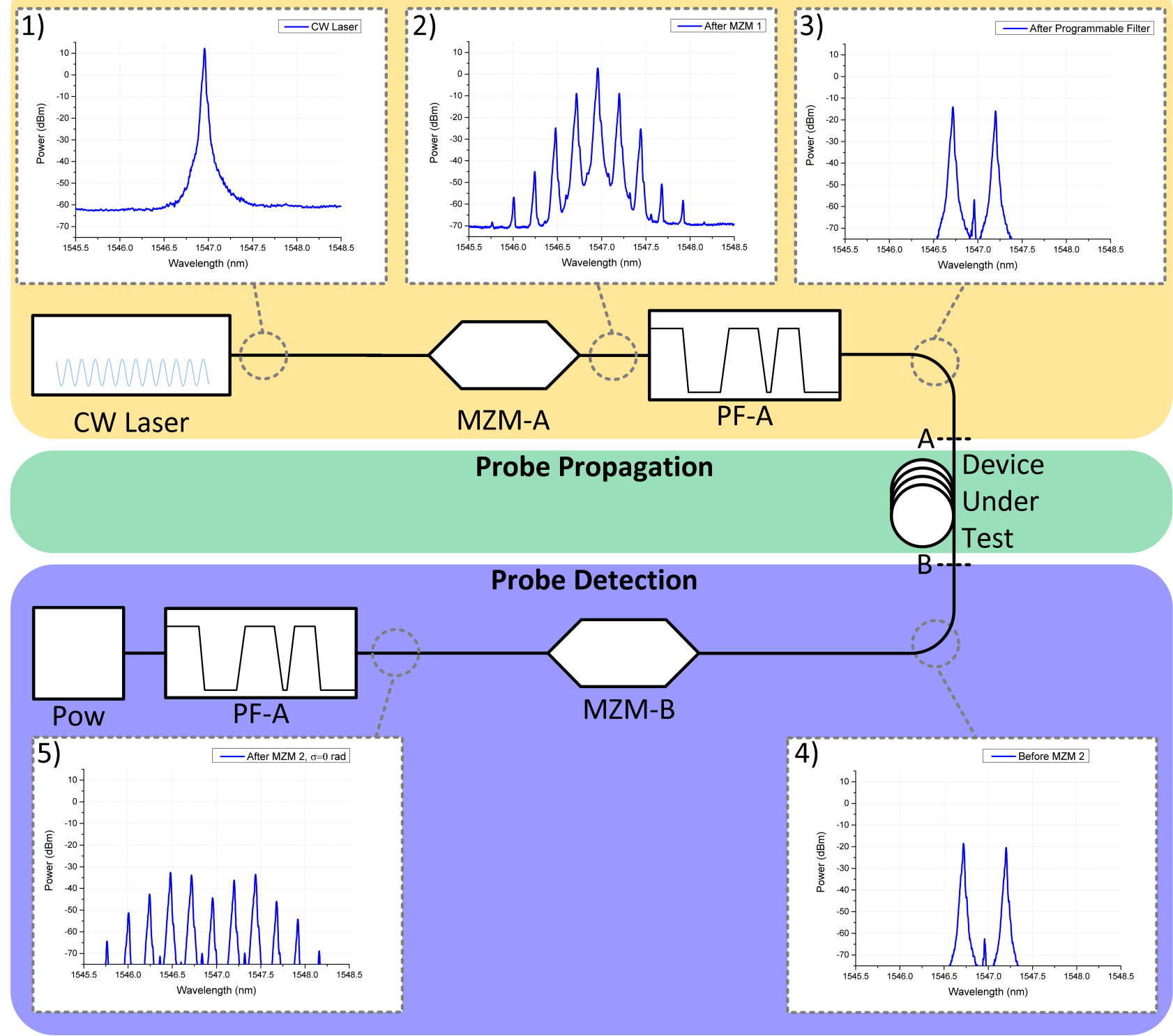

Figure 1 Archetypal experimental set-up. Spectra are provided at various stages of the system.

following relations:

$$
\begin{aligned}
\left|\psi_{\sigma=0}\right|^{2} & =\left[B_{+}^{\prime 2}+B_{-}^{\prime 2}+2 \cos \left(\Phi_{t o t}\right)\right] \\
\left|\psi_{\sigma=\frac{\pi}{2}}\right|^{2} & =\left[B_{+}^{\prime 2}+B_{-}^{\prime 2}+2 \sin \left(\Phi_{t o t}\right)\right] \\
\left|\psi_{\sigma=\pi}\right|^{2} & =\left[B_{+}^{\prime 2}+B_{-}^{\prime 2}-2 \cos \left(\Phi_{t o t}\right)\right] \\
\left|\psi_{\sigma=\frac{3 \pi}{2}}\right|^{2} & =\left[B_{+}^{\prime 2}+B_{-}^{\prime 2}-2 \sin \left(\Phi_{t o t}\right)\right]
\end{aligned}
$$

where $\Phi_{t o t}=\phi_{-}-\phi_{+}+\theta\left(\omega_{-}\right) L-\theta\left(\omega_{+}\right) L+\phi_{-}^{\prime}(f)-\phi_{+}^{\prime}(f)$ is the phase difference between the two tones. It is straight- forward to see that $\Phi_{t o t}$ can be obtained by:

$$
\Phi_{t o t}=\arctan \left\{\frac{\left|\psi_{\sigma=0}\right|^{2}-\left|\psi_{\sigma=\pi}\right|^{2}}{\left|\psi_{\sigma=\frac{\pi}{2}}\right|^{2}-\left|\psi_{\sigma=\frac{3 \pi}{2}}\right|^{2}}\right\}
$$

In other words, we can perform a measurement of the phase difference between the two tones by successively applying one of four phase shifts to one of the tones before interference and each time measuring the power of an interfering tone. Approaches using successive power measurements of the interference whilst shifting the phase of one tone are some times referred to as phase-shift interferometry [1]. As can be seen, we require no information about the underlying 


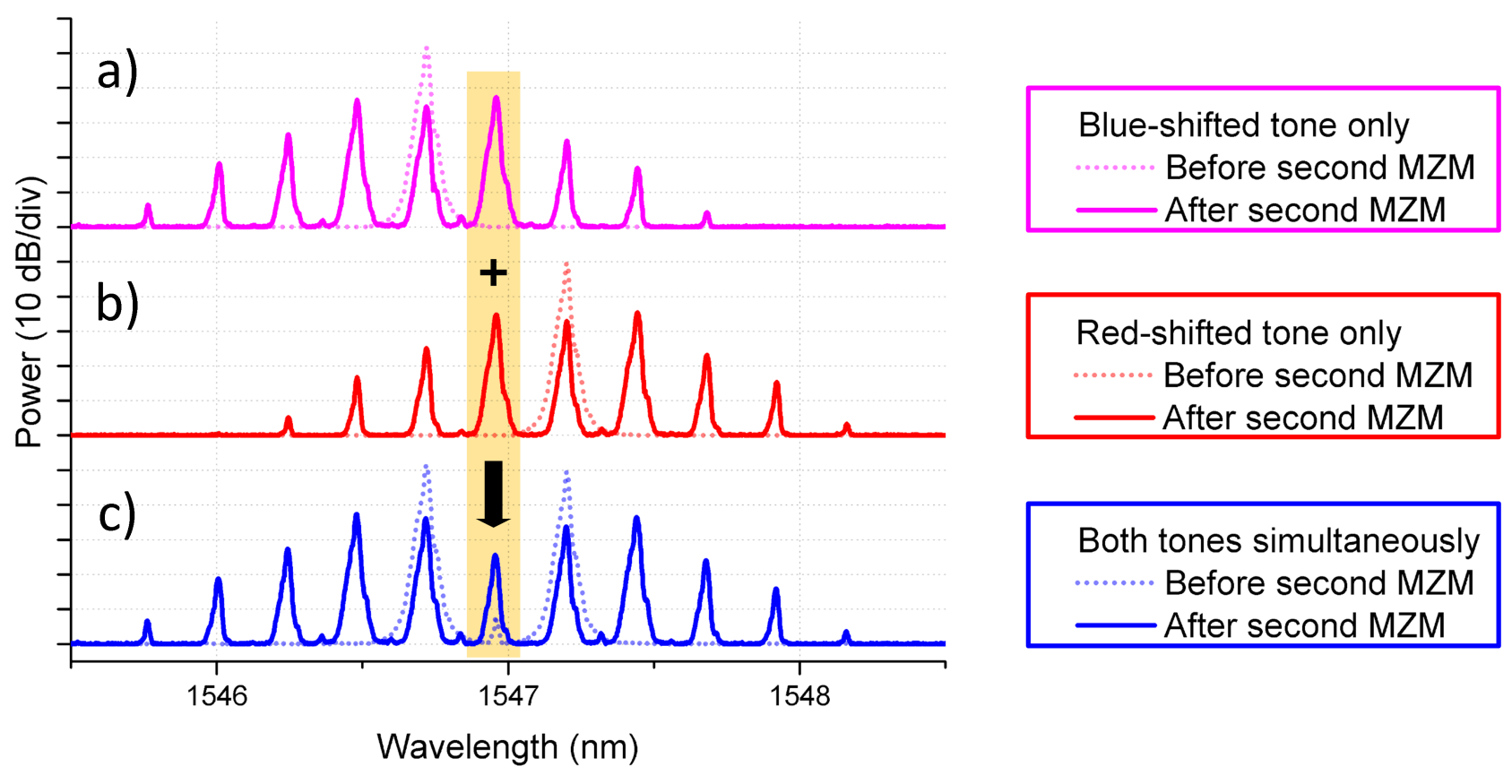

Figure 2 Spectra showing before (dotted line) and after (solid line) the second Mach-Zehnder modulator for the following scenarios: a) blue-shifted tone only, b) red-shifted tone only c) interefence resulting from the modulation of both tones.

relative powers of the two tones, which proves that not only can we tolerate variations in tone power (provided that this is over time scales longer than that taken for the four measurements given in Equations 5a, 5b, 5c and 5d), but also that we may apply the scheme to devices with wavelength dependent loss with no modification. An additional benefit of this approach compared to taking a simple interferogram is that the accuracy of each phase measurement is the same regardless of the phase difference between the measured tones. In contrast, regions of an interferogram with low power (due to destructive interference) are of compromised accuracy relative to higher power regions (formed from constructive interference) because of their lower signal to noise ratio, due to, amongst other things, shot noise, dark current etc. If further accuracy is desired, more values of $\sigma$ can be used and the resulting power of the interference measured. It can be seen that such a measurement effectively traces out a single sinusoid as the two tones pass between constructive and destructive interference, and it is the phase of this sinusoid which indicates the relative phase of the two tones.

\section{Applications}

At this point, measurement of the phase difference between the two tones is complete. In this section, we will discuss a number of applications of the spectral difference interferometry scheme. There are clearly many applications of a scheme which can measure the phase difference between two tones. In this work, we demonstrate the scheme's performance when it is applied to three tasks: the measurement of chromatic dispersion, analysis of the phase transfer pro- file of a ring resonator and finally, the determination of the nonlinear coefficient of an optical fibre.

As the first two applications (the measurement of chromatic dispersion and analysis of the phase transfer profile of a ring resonator) depend upon wavelength, we must naturally measure the phase difference between the two tones across the wavelength region of interest. To do this, we sweep the frequency of the original carrier, $f_{c}$ in order to sample the function $\Phi_{\text {tot }}\left(f_{c}, f_{s}\right)$, which we shall refer to as the phase difference function. As $\phi_{-}^{\prime}(f)$ and $\phi_{+}^{\prime}(f)$ depend only upon the experimental set-up and not the DUT, a measurement of $\Phi\left(f_{c}\right)$ without the DUT installed effectively constitutes a measurement of the residual dispersion of the measurement device. By subtracting the measurement of the residual phase difference function, $\Phi_{\text {res }}=\phi_{-}^{\prime}(f)+\phi_{+}^{\prime}(f)$, from the measurement taken with the DUT in place, Eq. 6 , we can obtain the actual phase difference function of the DUT, which can be seen to be equal to:

$$
\begin{aligned}
\Phi_{\mathrm{DUT}}\left(f_{c}, f_{s}\right) & =\Phi_{\mathrm{tot}}\left(f_{c}, f_{s}\right)-\Phi_{\mathrm{res}}\left(f_{c}, f_{s}\right) \\
& =\theta\left(\omega_{-}\right) L-\theta\left(\omega_{+}\right) L
\end{aligned}
$$

Once the phase difference function of the DUT, $\Phi_{\text {DUT }}\left(f_{c}, f_{s}\right)$, has been obtained, it may be used to obtain a number of parameters of the device.

\subsection{Chromatic Dispersion}

In the context of chromatic dispersion, we can see that $\theta(\omega)$ is more commonly represented by $\beta(\omega)$, the propagation constant. The chromatic dispersion of the device can be 
simply obtained using the following relationship between dispersion and propagation constant [4]:

$$
D=\frac{\mathrm{d}}{\mathrm{d} \lambda}\left(\frac{\mathrm{d} \beta}{\mathrm{d} \omega}\right)
$$

Combining Equations 8 and 9, we may obtain an approximation to the first derivative of $\beta\left(\omega_{c}\right)$ :

$$
\frac{\mathrm{d} \beta\left(\omega_{c}\right)}{\mathrm{d} \omega} \approx \frac{\Delta \beta\left(\omega_{c}\right)}{\Delta \omega}=\frac{\Phi_{D U T}\left(f_{c}, f_{s}\right)}{L\left(\omega_{-}-\omega_{+}\right)}
$$

Eqn. 10 can be seen to be an instance of the central difference derivative method [5] and hence has an error order of $\mathscr{O}\left(f_{s}^{2}\right)$. Naturally, decreasing the size of $f_{s}$ should be expected to increase the accuracy of the approximation. In our implementation, the smallest size of $f_{s}$ achievable was limited by the spectral resolution of the programmable filter to $10 \mathrm{GHz}$, but other implementations can offer smaller spacings. We note that there are other approaches to reducing this error, such as Richardson Extrapolation [6,7], to scalably reduce the error of the estimate of Eqn. 9. Although reducing $f_{S}$ decreases the error in the derivative approximation, there can be, in general, lower bounds to $f_{S}$ beyond which further reduction of $f_{S}$ will increase uncertainty in the determination of $\beta\left(\omega_{C}\right)$. This becomes clear when we consider the non-zero linewidth of $f_{S}$ (due to a nonmonochromatic RF source driving the modulators) which creates variations in our exposure to $\beta(\omega)$ over time. This error depends upon the relative size of the frequency separation to this linewidth and so should grow as $\propto \frac{2 d f_{S}}{f_{S}}$. Once we have sampled Eqn. 10 for a range of values of $\lambda$, we can perform the differentiation with respect to $\lambda$. Eqn. 10 can then be substituted into Eqn. 9, yielding an approximation for the chromatic dispersion of the DUT:

$$
D=\frac{\mathrm{d}}{\mathrm{d} \lambda}\left(\frac{\Phi_{D U T}\left(f_{c}, f_{s}\right)}{L\left(\omega_{-}-\omega_{+}\right)}\right)
$$

\subsubsection{Results}

With the measurement procedure described, we now proceed to present a number of proofs of the functioning of the scheme (some of which were first reported in [8]). We can split these measurements into two groups, those made on devices long enough to challenge typical Mach-Zehnder based interferometric schemes, and those made on devices short enough that their net dispersion is below the sensitivity of typical envelope detection based methods. We start by performing a series of measurements on fibres with weak birefringence, for which we make a small alteration to the experimental set-up as shown in Figure 3, resulting in the set-up shown in Figure 3, but other than this, the functioning remains unchanged. We use a Faraday mirror after the DUT in order for the signals to make a second pass through the DUT, but with an orthogonal polarisation to the first pass. This allows us to measure the mean dispersion of the fast and slow axes of the fibre, which is a more meaningful measure for low-birefringence fibres exhibiting strong coupling between polarisation modes. The first measurement to be presented was performed on $200 \mathrm{~m}$ of dispersion flattened highly nonlinear fibre (HNLF). The processing procedure is illustrated explicitly in Figure 4. Firstly, $\Phi_{\text {tot }}\left(f_{c}, f_{s}\right)$ is measured with the DUT installed (orange triangles, Figure 4-a) before a quadratic polynomial is fit to the resultant data (blue line, Figure 4-a). A second measurement and quadratic fit are performed with the DUT removed from the system, resulting in the orange triangles and blue line of Figure 4-b, respectively, providing a measurement of the residual phase difference function of the system, $\Phi_{r e s}\left(f_{c}, f_{s}\right)$. Subtracting the polynomial fit in Figure 4-b from that in Figure 4-a, we obtain the curve in Figure 4-c, the actual phase difference function of the DUT, $\Phi_{D U T}\left(f_{c}, f_{s}\right)$. This curve can then be differentiated with respect to wavelength and scaled according to Equation 10 to obtain the dispersion curve provided in Figure 4-d, plotted with a solid black line. As can be seen, as we fit the curve with a quadratic equation, we obtain a linear approximation to dispersion in this region. If knowledge of higher order dispersive effects is desired, the raw data can instead be fitted with higher order polynomials. Alongside the results obtained using the proposed measurement scheme, we also provide a plot of the very same fibre measured using the well-known differential phase method [9] (see also standard TIA-455-175 [10]). We can see a very good agreement between the two techniques, proving comparability between the two schemes for such net dispersions. Moving on, we now apply the proposed technique to the measurement of $1 \mathrm{~km}$ of standard telecommunications fibre (SMF-28). Measuring such a length of fibre, which has a net dispersion of $\approx 17 \times 1=17 \mathrm{ps} \mathrm{nm}^{-1} \mathrm{~km}^{-1}$, would present a challenge if undertaken using a Mach-Zehnder interferometric scheme, requiring a very long delay line to provide a reference wave. Because of this, many interferometric measurements of the chromatic dispersion of fibre are performed on very short lengths (at most in the metres regime) of the sample $[11,12]$. As such, measurements of such short samples can only give an estimate of the actual dispersion of the full length of test fibre meaning direct measurements must be used if accuracy is important. Figure 5 shows the results of this measurement (solid orange line) plotted alongside an empirical formula (dashed blue lines) provided by Corning to estimate the chromatic dispersion of SMF-28: $D_{28}(\lambda) \approx \frac{S_{0}}{4}\left(\lambda-\frac{\lambda_{0}^{4}}{\lambda^{3}}\right)$, where $S_{0} \leq 0.092 \mathrm{ps} \mathrm{nm}^{-1} \mathrm{~km}^{-1}$ and $1302 \mathrm{~nm} \leq \lambda_{0} \leq 1322 \mathrm{~nm}$. Using $S_{0}=0.092 \mathrm{ps} \mathrm{nm}^{-1} \mathrm{~km}^{-1}$ and $\lambda_{0}=1308 \mathrm{~nm}$, we once again see a strong agreement between the two plots, further confirming the correct functioning of the scheme.

We now consider devices with much lower net dispersions of $<0.2 \mathrm{ps} \mathrm{nm}^{-1} \mathrm{~km}^{-1}$. Firstly, we consider short lengths of SMF-28, specifically patchcords of length $1 \mathrm{~m}$, $3.1 \mathrm{~m}$ and $6.1 \mathrm{~m}$. As is well-known, in general, optical fibre exhibits longitudinal variations in dispersion, variations which can occur over metre-long length scales [13]. Hence, it is unreasonable to expect that measurements on such short lengths of fibre will agree with the nominal values of bulk SMF-28. We therefore resort to a self-referencing measurement, performing measurements on the afformentioned fibre and various concatenations thereof. The 6 measure- 


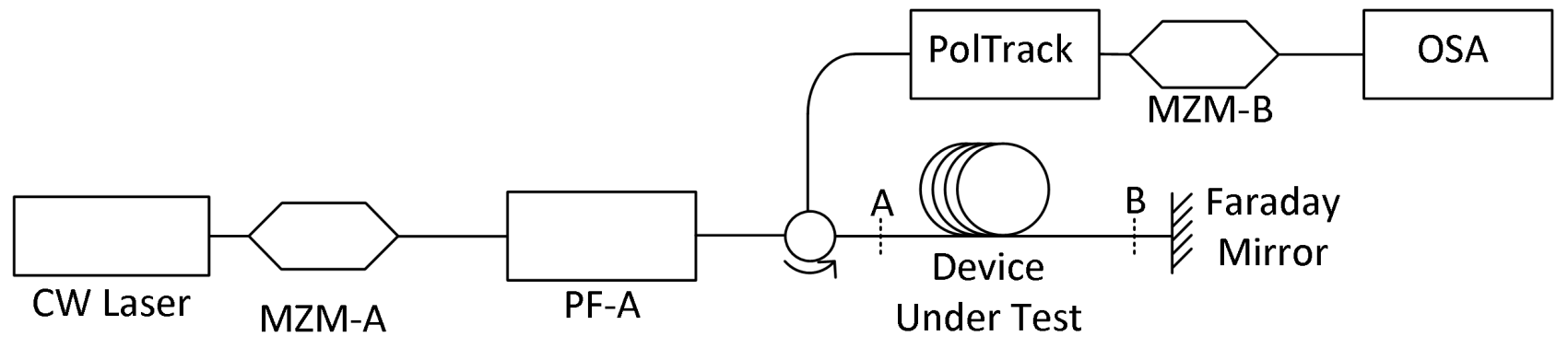

Figure 3 Modified experimental set-up for fibres with low birefringence. Note the incorporation of a circulator and a Faraday mirror in order for the probe tones to make a double pass through the fibre.
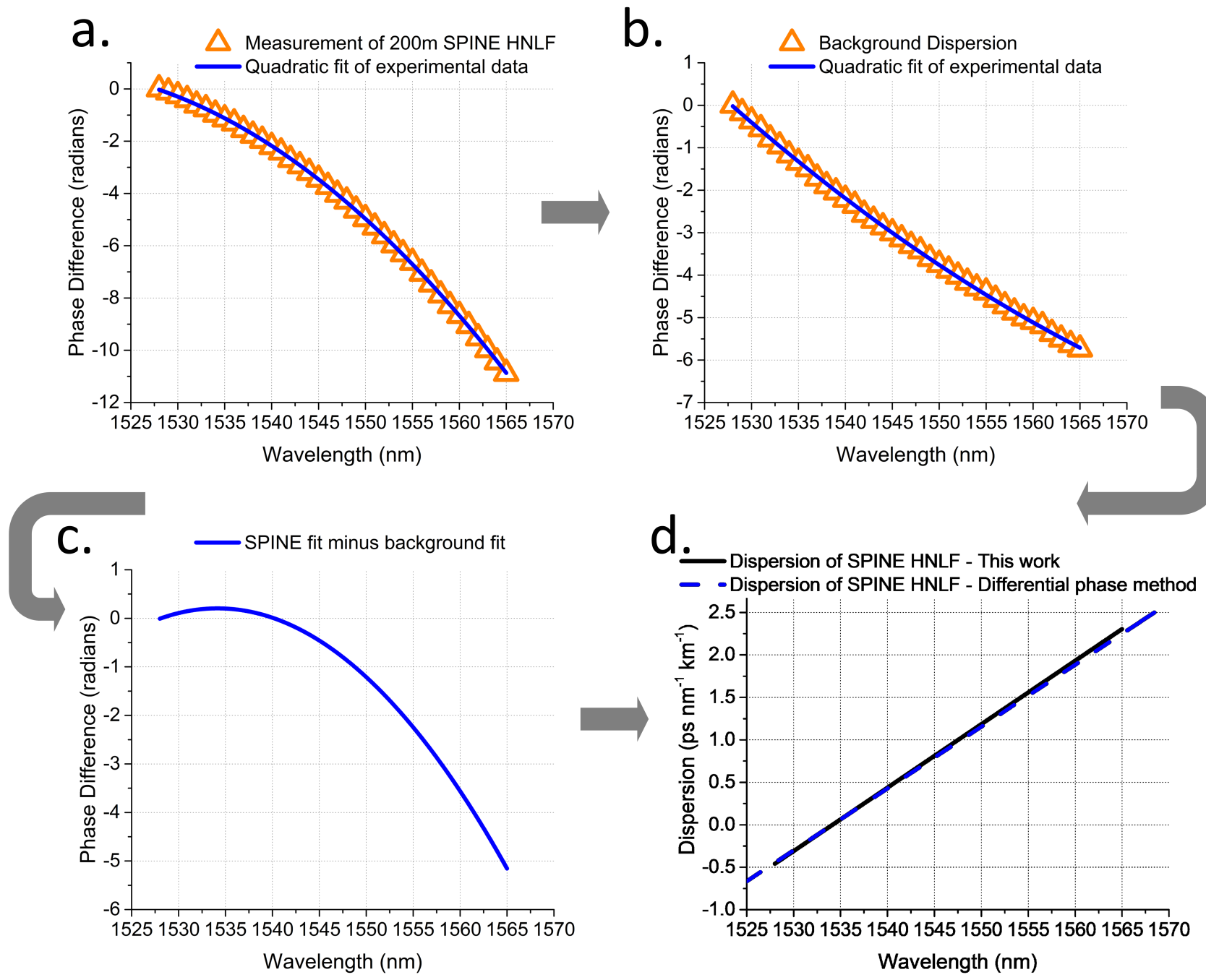

Figure 4 Processing steps to obtain an estimate for chromatic dispersion from the raw phase difference function measurements. a) Raw measurement of the DUT is fit with a quadratic polynomial; b) Raw measurement of the residual dispersion (with the DUT removed) is fit with another quadratic polynomial; c) subtraction of b) from a); d) rescaling and differentiation of d) results in the dispersion curve plotted with the solid black line, the results of the differential phase method (see text) are plotted with a dashed blue line for comparison. 
ment scenarios considered are shown in Table 1. If we have performed an accurate measurement of, for instance, M1, we should find that M1 + M5, which is equivalent to a total fibre length of $10.2 \mathrm{~m}$, should be in agreement with the direct measurement of M6 itself. Following this principle, we consider the measurement combinations: M6, M1 + M5, $\mathrm{M} 2+\mathrm{M} 4$ and $\mathrm{M} 1+\mathrm{M} 2+\mathrm{M} 3$, all of which should result in an estimate for dispersion equivalent to the full $10.2 \mathrm{~m}$ of fibre formed when all three patchords are connected together. The net dispersions of these combinations are plotted in Figure 5 wherein very good agreement can be seen between all of the combinations.

Next we quantify the repeatability of the scheme through the standard deviation of successive dispersion measurements on the same fibre. This study was first performed for an DCF fibre of length $47 \mathrm{~m}$ (with a net dispersion of $4.3 \mathrm{ps} \mathrm{nm}^{-1}$ at a wavelength of $1545 \mathrm{~nm}$ ). As always, a background measurement is essential, and so we take a large number of measurements of the background differential phase equation of the measurement system, in this case 150 , and average all of them together. A large number of measurements was taken to reduce the uncertainty in the background measurement. For the $47 \mathrm{~m}$ of DCF, we need not have as many measurement of the differential phase equations, and in this case acquired 43. The procedure described in Figure 4 was performed for each of the 43 DCF measurements, resulting in 43 error curves. Figure 6 provides the results of this analysis; in blue is plotted the mean of all of the 43 obtained dispersion measurements, whilst the dashed lines indicate the range of one standard deviation of the dispersion measurement about the mean. The standard deviation can be seen to be a pretty constant $0.06 \mathrm{ps} \mathrm{nm}^{-1} \mathrm{~km}^{-1}$ across the measured wavelength range.

\subsection{Phase Shift}

In addition to using the differential phase shift function to derive chromatic dispersion through differentiation, we may also determine the frequency dependent net phase shift resulting from propagation through a device. We make use of Equation 10, once again, but this time multiply through by $L$ :

$$
\frac{\mathrm{d} \theta\left(\omega_{c}\right)}{\mathrm{d} \omega} L \approx \frac{\Phi_{D U T}\left(f_{c}, f_{s}\right)}{\left(\omega_{-}-\omega_{+}\right)}
$$

Integrating the left hand side of Equation 12 over $\omega$ yields:

$$
\int \frac{\mathrm{d} \theta\left(\omega_{c}\right)}{\mathrm{d} \omega} L d \omega=\theta\left(\omega_{c}\right) L+c=\phi\left(\omega_{c}\right)+c
$$

which, returning to Equation 12, indicates that:

$$
\phi\left(\omega_{c}\right)+c \approx \int \frac{\Phi_{D U T}\left(f_{c}, f_{s}\right)}{\left(\omega_{-}-\omega_{+}\right)} d \omega+c
$$

In other words, the wavelength dependent phase shift caused by a device (plus some constant) can be obtained by integration of the phase difference function. As we are interested in the relative phase shift across the band, and not the absolute optical path length, we may safely ignore this constant and set it to zero.

\subsubsection{Results}

We apply the technique above to the measurement of the phase response of a ring resonator because of its strong but well-studied phase and amplitude transfer characteristics. The ring is a grating coupled, silcon structure, fabricated using a CMOS compatible process. More details of the ring, including its design, further characterisation and application to the generation of optical frequency combs can be found in [14]. The operating regime of ring resonators is generally split into three categories: undercoupled when the roundtrip loss is greater than the cross-coupling ratio, overcoupled when the roundtrip loss is less than the cross-coupling ratio, and critically-coupled when roundtrip loss is equal to the cross-coupling ratio. Control of the PN junction bias allows the coupling regime to be controlled [15].

Figure 7 shows the procedure by which we obtain the phase transfer profile of the ring resonator. Figure 7-a shows in orange the measured response of a straight, reference waveguide (which naturally includes the residual dispersion of the measurement set-up), whilst the plot in blue shows the response of the ring itself. After subtraction of the straight waveguide response from the ring resonator, the data is rescaled by division by $2 \pi \times 60 \times 10^{9}$ (the radial frequency spacing of the two probe tones), and shifted such that the broad peaks of the curve lie at a phase difference of zero, we obtain the plot in Figure 7-b. Knowing that the phase transfer profile of a ring resonator should consist of flattopped steps, we vertically align the peaks such that their tops lie at 0 . We finally integrate the curve in Figure 7-b over $\omega$, to obtain the actual phase transfer curve of the resonator shown in Figure 7-c.

An optical micrograph of the ring resonator is provided in Figure 8-a, for reference. As is known [15], the phase shift imparted by a ring resonator upon its input is associated with a concomitant modulation of the intensity of the output. Figure 8 provides 3 plots, the first, Figure $8-b$, shows a plot containing 4 resonances exhibited by the (overcoupled) ring resonator within the C-band. Each trough can be seen to coincide with a vertical step in the phase transfer function. We focus in on the resonance occurring about a wavelength of $1545 \mathrm{~nm}$, and apply an appropriate DC bias in order to measure the intensity and phase transfer functions of the ring in both the over- and undercoupling regimes. Figure 8$\mathrm{c}$ shows the response of the resonator with a forward bias voltage of $0.96 \mathrm{~V}$, in which case we can see that the system is in the overcoupled regime. Increasing the forward bias voltage further to $1.1 \mathrm{~V}$ increases the loss of the ring to the extent that it enters the undercoupled regime, as shown in Figure 8-d. 
B. R. H. Bottrill ${ }^{1,}{ }^{*}$, M. A. Ettabib ${ }^{1}$, I. Demirtzioglou ${ }^{1}$, R. Marchetti ${ }^{2}$, C. Lacava ${ }^{1}$, F. Parmigiani ${ }^{3}$,D.J. Richardson ${ }^{1}$ and P. Petropoulos ${ }^{1}:$ Spectral Differer

\begin{tabular}{c|c|c|c|c|c|c} 
Measurement & M1 & M2 & M3 & M4 & M5 & M6 \\
\hline \hline Combination & $1 \mathrm{~m}$ & $3.1 \mathrm{~m}$ & $6.1 \mathrm{~m}$ & $1+6.1$ & $3.1+6.1$ & $1+3.1+6.1$ \\
\hline Total Length & $1 \mathrm{~m}$ & $3.1 \mathrm{~m}$ & $6.1 \mathrm{~m}$ & $7.1 \mathrm{~m}$ & $9.2 \mathrm{~m}$ & $10.2 \mathrm{~m}$
\end{tabular}

Table 1 Fibre combinations.

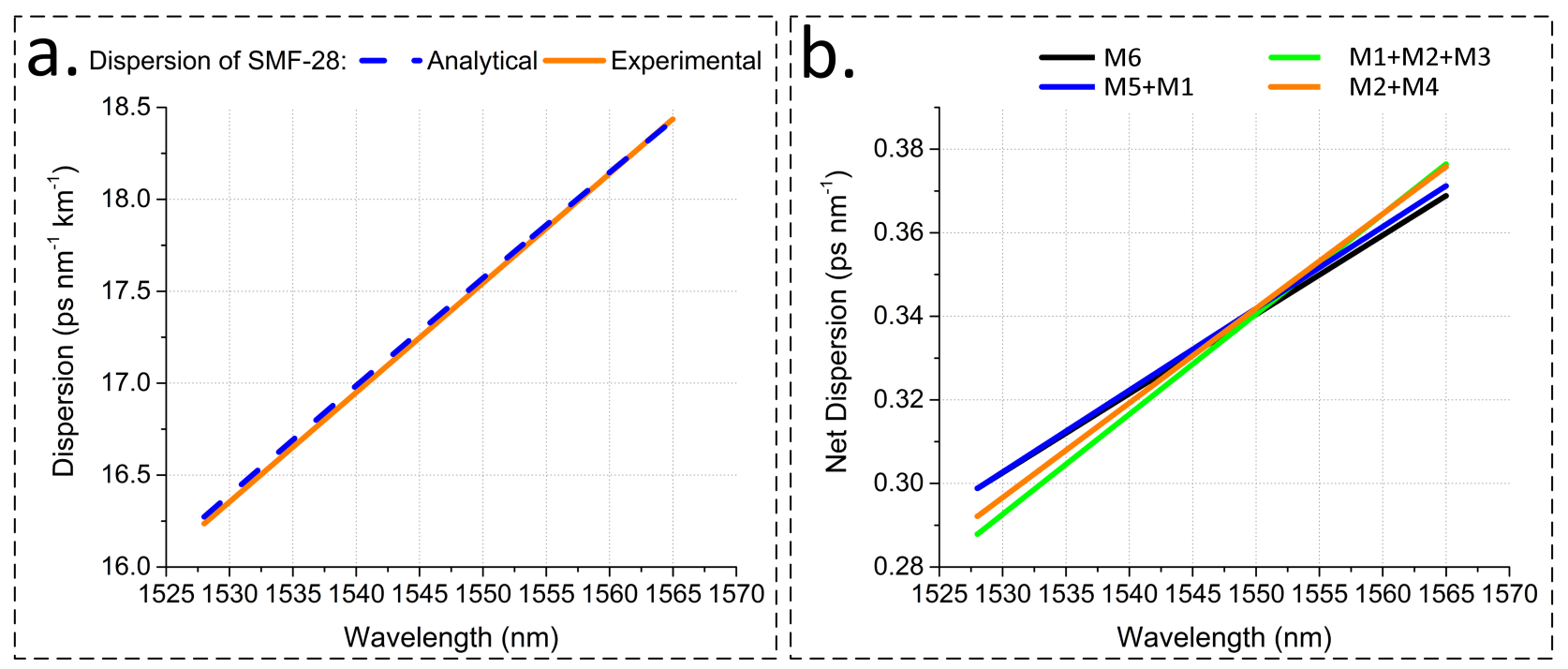

Figure 5 Results of dispersion measurements: a) $1 \mathrm{~km}$ of SMF-28 measured using the proposed scheme as well as the differential phase method. b) Combinations of patchords with length totalling $10 \mathrm{~m}$.

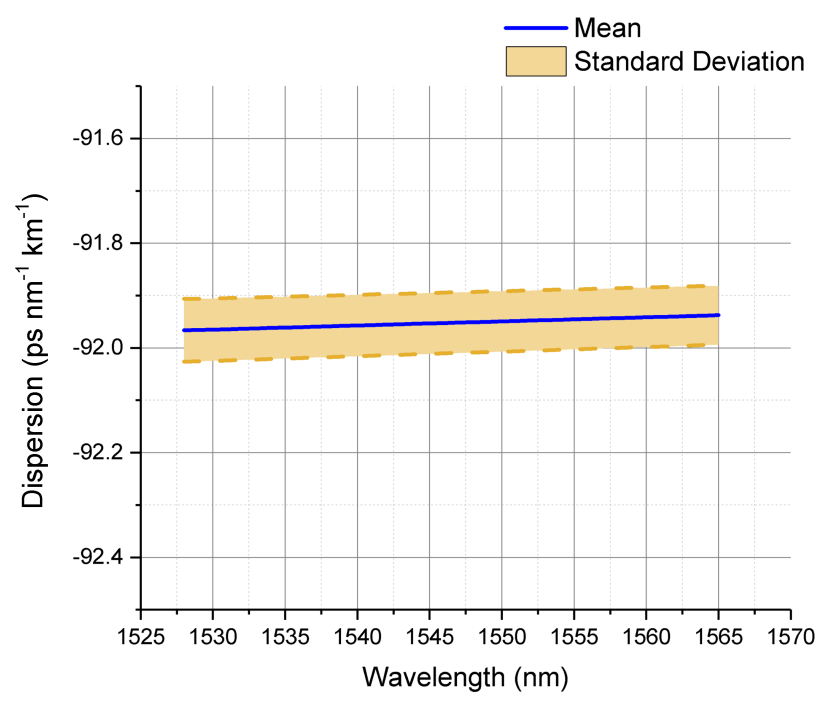

Figure 6 Estimate of the chromatic dispersion of a 200m length of SMF-28e obtained by averaging 100 underlying measurements. The mean of this measurement is plotted with the solid blue line, whilst the standard deviation is indicated by the dashed orange line and orange shading.

\subsection{Nonlinearity}

We now present the application of our proposed scheme to the measurement of the nonlinear coefficient of a fibre. Al- though our main motivation in performing this measurement is to exhibit the versatility of the proposed measurement scheme, adopting this method does indeed present some advantages compared to, for instance, four-wave mixing based approaches to measuring a medium's nonlinear coefficient, namely, being unaffected by any dispersive effects in the medium. The mathematical relationship which allows us to derive the nonlinear coefficent of the medium can be obtained by considering the phase shift experienced by each of the two tones launched into the medium due to self-phase modulation (SPM) and cross-phase modulation (XPM):

$$
\begin{aligned}
& \phi_{-}=\underbrace{\gamma L P_{-}}_{\mathrm{SPM}}+\underbrace{2 \gamma L P_{+}}_{\mathrm{XPM}}+\phi_{-}^{\prime} \\
& \phi_{+}=\underbrace{\gamma L P_{+}}_{\mathrm{SPM}}+\underbrace{2 \gamma L P_{-}}_{\mathrm{XPM}}+\phi_{+}^{\prime}
\end{aligned}
$$

Subtracting Eq. 15 from Eq. 16 leads to:

$$
\Delta \Phi=\gamma L\left(P_{+}-P_{-}\right)+\phi_{-}^{\prime}-\phi_{+}^{\prime}
$$

i.e. we find that the phase difference between the two tones changes linearly with the nonlinear coefficient, $\gamma$, the length of the medium, $L$ and the linear power difference between the two tones. It is clear from Eq 17 that plotting $\Delta \Phi$ vs $P_{+}-P_{-}$results in a linear curve with gradient $\gamma L$ and hence we can obtain an estimate of $\gamma$. 

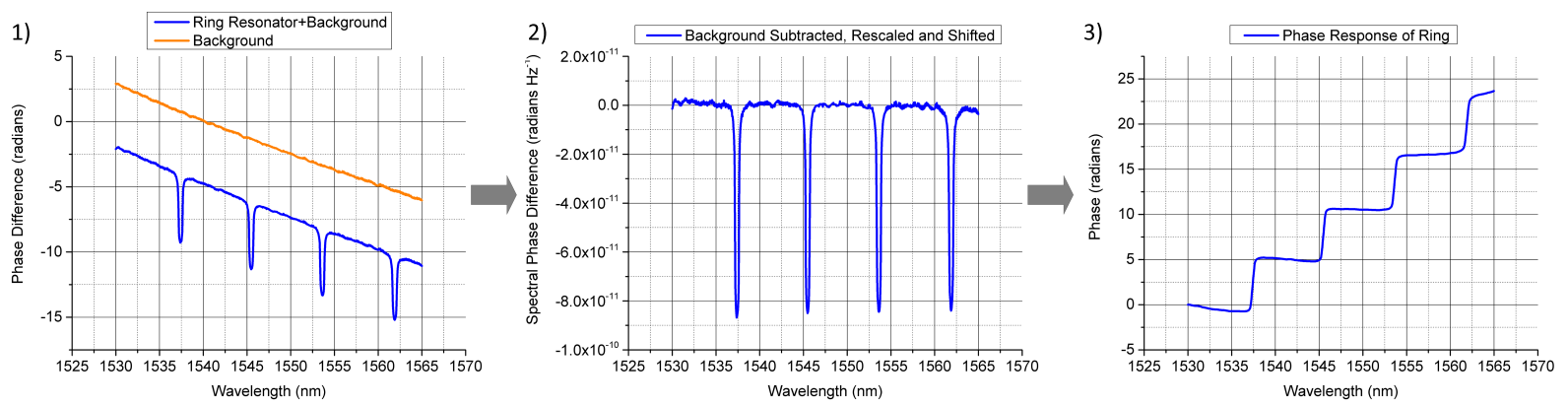

Figure 7 Processing procedure used to obtain the phase transfer function of the ring resonator from the phase difference function. 1) plots of raw measurement of DUT and the background measurement; 2) subtraction of the background measurement from the measurement of the DUT, flat-topped peaks shifted to $0 \mathrm{rad}$; 3) integration and rescaling of 2) leads to the characteristic staircase shaped phase transfer function of an overcoupled ring resonator.

a)

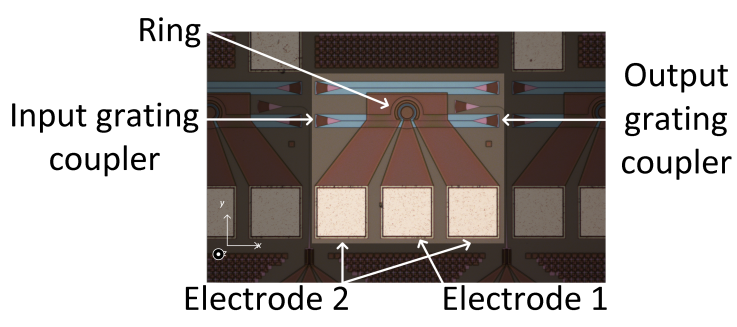

Overcoupled

c)

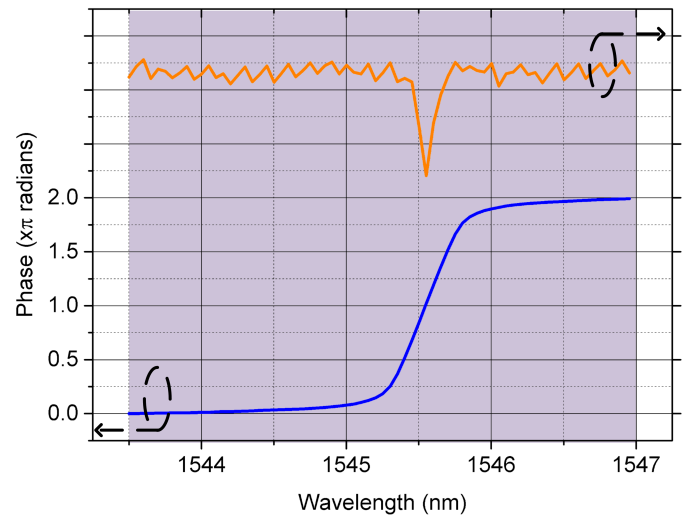

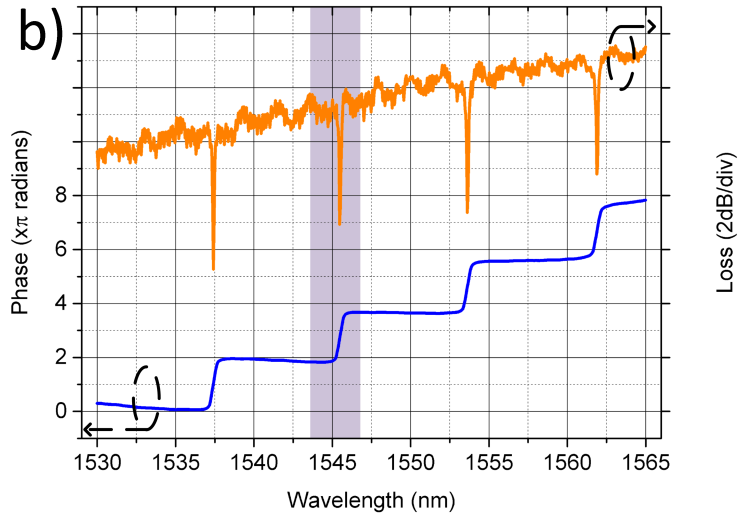

Undercoupled
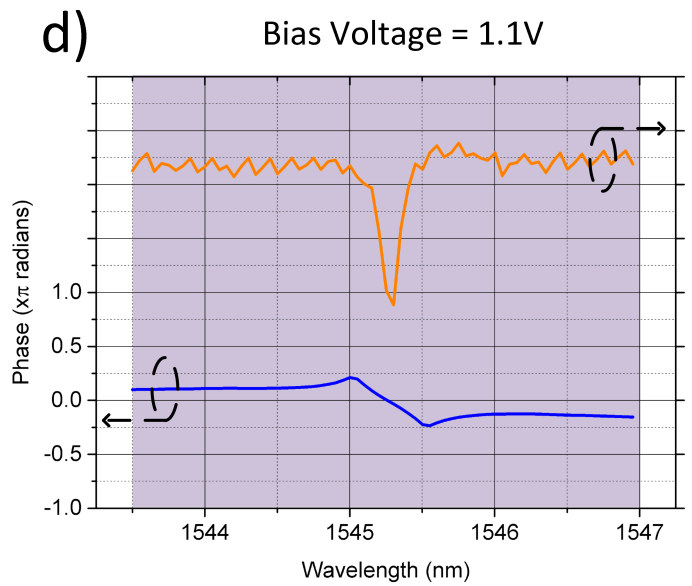

Figure 8 a) Optical micrograph of the ring resonator; b), c) d) provide plots of the phase transfer function (plotted in blue) and wavelength dependent loss (plotted in orange) of the ring resonator for different scenarios: b) full C-band view of the ring resonator in the overcoupled state; c) fine detail plot about the resonance at $1545 \mathrm{~nm}$ when the bias voltage is $0.96 \mathrm{~V}$ (leading to overcoupling); $\mathrm{d}$ ) fine detail plot about the resonance at $1545 \mathrm{~nm}$ when the bias voltage is $1.1 \mathrm{~V}$ (leading to undercoupling)

For further confirmation, we also estimate the nonlinear ing can be considered to be independent of the dispersion, coefficient by assuming that the evolution of four-wave mix- 
relying solely upon the nonlinear coefficient of the medium, a seemingly reasonable assumption seeing that the tones are spaced by only $60 \mathrm{GHz}$ and given that the fibre has low dispersion. This assumption allows us to use the method proposed by Boskovic et al [16], which makes use of the following analytical relation between the signal to 1st order idler power ratio:

$$
\frac{P_{-1}}{P_{1}}=\frac{\mathrm{J}_{0}^{2}\left(2 \sqrt{P_{0} P_{1}} \gamma L\right)+\mathrm{J}_{1}^{2}\left(2 \sqrt{\mathrm{P}_{0} P_{1}} \gamma L\right)}{\mathrm{J}_{1}^{2}\left(2 \sqrt{P_{0} P_{1}} \gamma L\right)+\mathrm{J}_{2}^{2}\left(2 \sqrt{P_{0} P_{1}} \gamma L\right)}
$$

where $P_{0}, P_{1}$ and $P_{-1}$ are the pump, signal and 1st order idler optical powers. To obtain an estimate for $\gamma$, we simply measure $P_{0}, P_{1}, P_{-1}$ and $L$, and search for the value of $\gamma$ which allows Equation 18 to be satisfied using a simple optimisation algorithm.

\subsubsection{Results}

We apply these two methods first to the measurement of a low dispersion highly nonlinear fibre of length $300 \mathrm{~m}$, attenuation $0.79 \mathrm{~dB} \mathrm{~km}^{-1}$, dispersion $-0.08 \mathrm{ps} \mathrm{nm}^{-1} \mathrm{~km}^{-1}$ at $1550 \mathrm{~nm}$ and dispersion slope $0.018 \mathrm{ps} \mathrm{nm}^{-2} \mathrm{~km}^{-1}$, leading to an effective length [4] of $L_{e f f}$ of $0.292 \mathrm{~km}$. Figure 9 provides a plot of $\Delta \Phi$ vs $P_{+}-P_{\text {- }}$ for this fibre, along with a linear fit whose gradient is $2.55 \mathrm{rad} \mathrm{W}^{-1}$, indicating a value for the nonlinear coefficient of $2.55 / 0.292=8.73 \mathrm{~W}^{-1} \mathrm{~km}^{-1}$. This value is in good agreement with that obtained using Boscovic's method of $8.42 \mathrm{~W}^{-1} \mathrm{~km}^{-1}$.

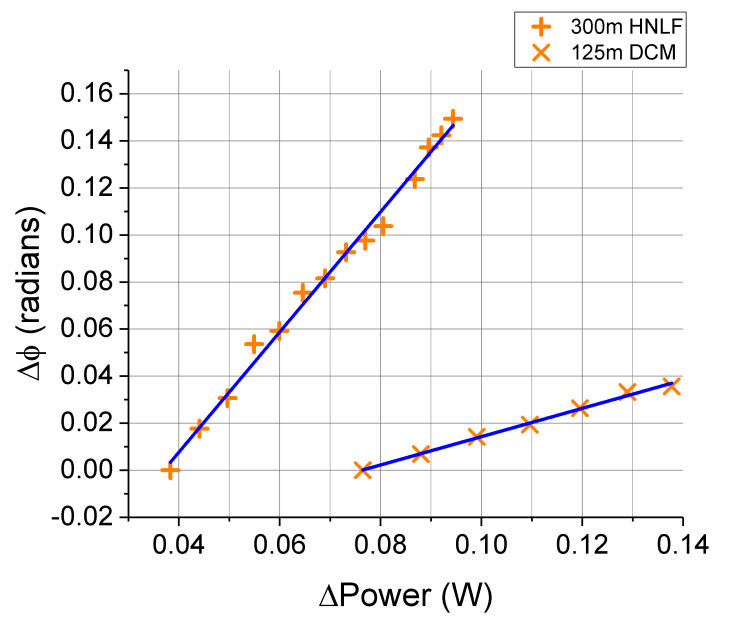

Figure 9 Plot of the phase difference between the two tones as it varies with the difference in power between them. Data is provided for an HNLF as well as a DCF. The gradients of these curves can be used to determine the nonlinear coefficients of each of the fibres.

We now consider the measurement of the nonlinear coefficient of dispersion compensating fibre. The dispersion compensating fibre used had a length of $125 \mathrm{~m}$, attenuation $0.442 \mathrm{~dB} \mathrm{~km}^{-1}$, dispersion $-106.49 \mathrm{ps} \mathrm{nm}^{-1} \mathrm{~km}^{-1}$ at
$1550 \mathrm{~nm}$ and dispersion slope $-0.392 \mathrm{ps} \mathrm{nm}^{-2} \mathrm{~km}^{-1}$, leading to an effective length [4] of $L_{e f f}=0.124 \mathrm{~km}$. As this is highly dispersive, it places a greater challenge upon the assumption that the fibre can be treated as dispersionless over the bandwidth considered. We should, therefore expect the efficiency of FWM in the fibre to be suppressed, leading to an underestimate of the magnitude of the nonlinear coefficient using Boscovic's method. In contrast, our proposed method, being insensitive to dispersion, should offer an accurate measurement of the nonlinear coefficient all the same. Figure 9 indeed shows this is the case, with Boscovic's method implying a smaller value of $\gamma$ of $3.86 \mathrm{~W}^{-1} \mathrm{~km}^{-1}$ compared to that obtained using our method of $0.60 / 0.124=4.85 \mathrm{~W}^{-1} \mathrm{~km}^{-1}$.

To support our attribution of the discrepancy between our method and Boscovic's method to the intolerability of the latter to dispersion, we performed some simple simulations using the commercial photonics simulation software, VPIphotonics DesignSuite. In the simulation, two CW tones are launched into fibre with parameters matching the HNLF and DCF listed above, with the values of the nonlinear coefficient being those obtained using our scheme, i.e. $\gamma_{H N L F}=8.73 \mathrm{~W}^{-1} \mathrm{~km}^{-1}$ and $\gamma_{D C F}=4.85 \mathrm{~W}^{-1} \mathrm{~km}^{-1}$. We extract the appropriate signal and idler powers from the simulated field and perform Boscovic's method to obtain the inferred nonlinear coefficient of each simulated fibre. For the simulated HNLF, we obtain $\gamma_{H N L F}=8.66 \mathrm{~W}^{-1} \mathrm{~km}^{-1}$ whilst for the simulated DCF, we obtain $\gamma_{D C F}=3.88 \mathrm{~W}^{-1} \mathrm{~km}^{-1}$, both in very good agreement to what we obtained experimentally, supporting our claim that the discrepency is due to intolerance of chromatic dispersion in Boscovic's method.

\subsection{Features of Spectral Difference Interferometry}

The factors limiting the accuracy of interferometric schemes are often practical ones - sensitivity to temperature variations, mechanical vibrations, limited wavelength tunability of laser sources etc. Indeed, many interferometric approaches to chromatic dispersion measurement, for instance, show only relatively small advantages compared to each other in terms of susceptibility to fundamental noise sources such as shot noise and thermal noise [17]. In this section, we will discuss a number of practical features possessed by our scheme.

We note that our scheme, with its single lightpath, shows very high immunity to mechanical vibration, allowing it to be implemented in optical fibre, deployed in mechanically harsh environments, and used to perform measurements on remote systems. This is in contrast to, for instance, the Mach-Zehnder topology, whose mechanical sensitivity dictates the adoption of increased complexity, for instance, fast sweeping lasers [18], phase dithering [19] or other forms of stabilisation feedback [20,21].

As discussed, many forms of interferometry utilise probe and reference tones directly sourced from a tunable laser, and hence the quality of this laser has a large impact on the accuracy of measurements. The bandwidth of these tones 
should be as small as possible and ideally, they should be monochromatic. In our scheme, the probe and reference tones are derived from the output of an optical frequency comb generated using a Mach-Zehnder modulator. The quality and stability of this comb, therefore, is important for determining the performance of the scheme. As the two tones are generated in the same modulator, drifts in the laser carrier frequency (which results in coherent broadening of the tone bandwidth) do not cause a frequency shift between the probe and reference tone (as they do in homodyne schemes), but rather result in a drift in relative phase shift due to the $\theta(\omega)$ of the DUT as well as potentially false attribution of a measurement to a particular wavelength. The impact of this effect upon our scheme relative to more typical, single tone intereformeters will depend upon the drift of the source laser and the gradient of $\theta(\omega)$ with $\omega$.

Due to propagation through the DUT, there is a time delay between the carrier passing through the first modulator and the probe and reference tone passing through the second. This means that any frequency jitter in RF tone driving the modulators (which leads to incoherent broadening of tone bandwidth) will directly result in a error in the phase measured by the scheme, as the comb formed by the second modulator will not have the exact same frequency spacing as the first, necessitating the use of a high quality clock source to drive the two modulators. For long duration measurements, it is necessary to ensure that the two modulators are sufficiently thermally stabilised, with temperature changes over the time duration of a single carrier frequency sweep $\left(f_{c}\right)$ being particularly harmful. In our demonstration, we utilised PID controlled thermoelectric coolers to stabilise the modulators, but approaches based on driving the DC bias port of the modulator are also possible.

Our proposed scheme requires only minimal numerical processing to extract the desired parameters, in contrast to fringe based white light [2] or sweeping arm [22] interferometry, but does, however, require four optical power measurements to generate one measurement of the relative phase between the two tones. The fast swept laser approach aims to overcome environmental error by performing a measurement over time scales faster than fluctuations in the environment. This makes the scheme essentially a synchronous method which requires the use of faster photodetection which comes at the price of reduced sensitivity. Our scheme permits accumulation of power measurements over longer periods of time, similar to classic homodyne detection methods, but without the need for complex stabilisation, facilitating higher sensitivity measurement.

Naturally, given the use of multiple tones in our scheme, performance is affected by the extinction ratio of the optical bandpass filters and modulators used. Other schemes have their own analogous issues, for instance, in general, Mach-Zehnder and Sagnac interferometers rely upon a constant coupling ratio between the probe and reference arms (or at least knowledge of any discrepancies). Although we found no challenge in meeting this requirement, we note that suppression of the residual carrier tone after the first Mach-Zehnder modulator did improve the performance of the scheme. Improving the performance of the scheme fur- ther will likely necessitate the use of higher extinction filtering, although we believe that higher extinction modulators would only be necessary for measurements in power limited scenarios.

\subsubsection{Advantages to the Measurement of Chromatic Dispersion}

Interferometric techniques are rarely used for measuring long lengths of fibre; this is something for which envelope detection schemes, such as the differential phase method [9], are often applied. We have shown in Figure 5 that our scheme may be applied to the measurement of net chromatic dispersions beyond $17 \mathrm{ps} \mathrm{nm}^{-1}$ (equivalent to $1 \mathrm{~km}$ of SMF28). Most interferometric schemes require the length of the reference arm to be matched to the length of the DUT in order to maximise the mutual coherence between the two tones or at least within the coherence length of the laser. This requirement is met either using a free-space reference arm or a fibre reference arm. In the former case, reference arms tend to become increasingly more challenging to construct with increasing length whilst in the latter case, long fibre lengths increase a system's susceptibility to mechanical and temperature fluctuations, not to mention the complication of having to adjust reference arm lengths to match different DUTs of potentially disparate length. Our scheme has no such issues, given that the probe and reference tones propagate in the same medium during testing. Further insight into the minimum and maximum measureable net dispersion by common interferometric schemes and our scheme can be obtained by considering the phase difference between two successive measurements. For a simple fringe-based interferometer this is given by:

$$
\Delta \Phi=L\left(\beta\left(\omega_{c}+\frac{\Delta \omega}{2}\right)-\beta\left(\omega_{c}-\frac{\Delta \omega}{2}\right)\right)
$$

where $\Delta \omega$ is the radial frequency step-size of the probe laser and $\omega_{c}$ is the radial frequency about which the two measurements are centred. The equivalent form for our proposed scheme, which essentially consists of the phase difference between two phase difference measurements, takes the form:

$$
\begin{aligned}
\Delta \Xi= & L\left(\left[\beta\left(\omega_{c}-\frac{\Delta \omega}{2}+\frac{\delta \omega}{2}\right)-\beta\left(\omega_{c}-\frac{\Delta \omega}{2}-\frac{\delta \omega}{2}\right)\right]\right. \\
& \left.-\left[\beta\left(\omega_{c}+\frac{\Delta \omega}{2}+\frac{\delta \omega}{2}\right)-\beta\left(\omega_{c}+\frac{\Delta \omega}{2}-\frac{\delta \omega}{2}\right)\right]\right)
\end{aligned}
$$

Where $\delta \omega$ is the radial frequency difference between the two probe tones. Assuming a truncated Taylor series expansion for the propagation constant, $\beta(\omega)=\beta_{0}+\beta_{1}(\omega-$ $\left.\omega_{0}\right)+\frac{1}{2} \beta_{2}\left(\omega-\omega_{0}\right)^{2}$, Equation 19 and Equation 20 can be simplified to become:

$$
\begin{aligned}
\Delta \Phi & =L \Delta \omega\left(\beta_{1}+\left(\omega_{0}-\omega_{c}\right) \beta_{2}\right) \\
\Delta \Xi & =L \Delta \omega \delta \omega \beta_{2}
\end{aligned}
$$


Equation 21 and Equation 22 expose a number of differences between typical Mach-Zehnder interferometer and our proposed interferometer. Firstly, we recall that in both cases there is an upper limit to the measureable net chromatic dispersion, determined by the maximum tolerable phase shift of each scheme as well as a lower limit, determined by the sensitivity of each scheme to small phase shifts.

The upper limit is determined by the maximum change in phase per frequency step, $\Delta \omega$, which can be measured by each scheme. For instance, given the modulo $2 \pi$ nature of phase arithmetic, the phase $\alpha$ rad is not distinguishable from $\alpha+2 \pi \mathrm{rad}$. If these phase step limits are exceeded, then we effectively observe aliasing. As can be seen in Equation 21, the phase shift between successive measurements derives from a combination of the group velocity, $\beta_{1}$, as well as the group velocity dispersion, $\beta_{2}$, (as well as higher order terms, $\beta_{3}, \beta_{4}$, etc). If information about the group velocity is not desired, the maximum tolerable phase shift is needlessly being expended through exposure to the group velocity term. In contrast, as can be seen from Equation 22, our proposed scheme has no dependence upon $\beta_{1}$, and so represents a more direct measurement of chromatic dispersion and higher order terms. The lack of exposure to $\beta_{1}$ increases the maximum chromatic dispersion which can be measured.

The phase shift in both schemes can be seen to be linearly proportional to $\Delta \omega$ and so by decreasing this step-size, we can decrease the corresponding values of $\Delta \Phi$ and $\Delta \Xi$, permitting for example, the measurement of larger values of net chromatic dispersion, $\beta_{2} L$. Inversely, increasing $\Delta \omega$ increases $\Delta \Phi$ and $\Delta \Xi$ increasing the sensitivity of the scheme to smaller values of $\beta_{2} L$. For schemes based upon a tunable laser, this approach depends heavily upon the wavelength tunability of the laser, whilst for spectral approaches, the limiting factor is the resolution of the spectrometer. Equation 22 shows that our scheme offers another degree of freedom, the probe tone spacing $\delta \omega$. This parameter can be varied with much the same effect as $\Delta \omega$, offering greater flexibility. This allows the trade-off between sensitivity and dispersion tolerance to be tuned independently of the wavelength tunability of the laser and the selected radial frequency step-size, $\Delta \omega$.

\subsubsection{Advantages to the Measurement of Phase Transfer Functions}

With respect to the measurement of the phase transfer profile of integrated photonic devices, the scheme also possesses a number of advantages relative to, for instance, the MachZehnder approach. The ring resonator we characterised possesses both an amplitude and phase transfer function. For simple interferogram based schemes, for instance, it is difficult to identify whether changes in the power of the interference are due to phase or amplitude changes in the device. This will either increase measurement uncertainty, or at the least, require further post-processing. Given our scheme's full-quadrature measurement, the scheme is agnostic to wavelength dependent attenuation. Furthermore, the lack of a reference arm means that remote devices located over distances greater than the coherence length of the probe laser can be measured, a notable advantage for sensing.

\subsubsection{Advantages to the Measurment of the Nonlinear Coefficient}

Finally, as evidenced in Section 3.3.1, the scheme allows us to directly measure the phase shift between two tones whilst being insensitive to dispersion. This stands in contrast to, for instance, the commonly used Boscovic method. We note that Mach-Zehnder approaches to the measurement of the nonlinear coefficient are also unaffected by the dispersion of the device, but again require some method of decoupling of amplitude and phase changes [23].

\section{Conclusion}

We have described and implemented a novel interferometric scheme applicable to a wide range of characterisation and monitoring tasks. To illustrate its versatility, we have applied it to the measurement of chromatic dispersion on SMF-28 with lengths ranging from 10 s of centimeters to kilometres. We then applied this method to the characterisation of the phase response of a ring resonator, and experimentally identified the phase shift associated with undercoupling and overcoupling regimes of a ring resonator. Finally, we have utilised the scheme to measure the nonlinear coefficient of some example fibres, showing that it offers advantages over, for instance, Boscovic's method in being insensitive to the dispersion of the device. Beyond this, it can be seen that the scheme can be modified to perform optical time domain reflectometry, allowing for the characterisation of optical properties along the length of a medium or used to measure the temperature or strain of a device. Indeed, when viewed as a novel interferometer, there is a large range of phenomema to which the scheme can be applied. The scheme shows excellent isolation from mechanical variations, with the probe and reference tones travelling in the same medium at all times, as well as independent control over the scheme's sensitivity by tuning of the tone spacing. Given that the scheme can be constructed using relatively standard optical telecommunications equipment, resulting in a robust system with high sensitivity, we believe that it is an attractive option when considering new measurement requirements.

\section{Acknowledgements}

The data for this work is accessible through the University of Southampton Institutional Research Repository (DOI:10.5258/SOTON/D1009). This work was supported by the following UK EPSRC grants: EP/S002871/1 as part of the Photonic Phase Conjugation Systems (PHOS) project and NS/A000021/1, the National Dark Fibre Infrastructure Service. 


\section{References}

[1] P. Hariharan, Basics of Interferometry (Elsevier Science, 2010).

[2] J. Y. Lee and D. Y. Kim, Opt. Express 14(24), 11608-11615 (2006).

[3] T. Sakamoto, T. Kawanishi, and M. Izutsu, Optimization of electro-optic comb generation using conventional machzehnder modulator, in: Microwave Photonics, 2007 Interntional Topical Meeting on, (Oct 2007), pp. 50-53.

[4] G. P. Agrawal, Nonlinear fiber optics (Elsevier/Acad. Press, Amsterdam, 2007).

[5] P. O. J. Scherer, in: Numerical Differentiation (Springer International Publishing, Heidelberg, 2013), pp. 37-43.

[6] L. F. Richardson, Philosophical Transactions of the Royal Society of London A: Mathematical, Physical and Engineering Sciences 210(459-470), 307-357 (1911).

[7] P. DeVries and J. Hasbun, A First Course in Computational Physics (Jones \& Bartlett Learning, 2011).

[8] K. Bottrill, M. Ettabib, J. Gates, C. Lacava, F. Parmigiani, D. Richardson, and P. Petropoulos, Flexible scheme for measuring chromatic dispersion based on interference of frequency tones, in: Optical Fiber Communication Conference, (Optical Society of America, 2017), p. Th4H.5.

[9] B. Costa, D. Mazzoni, M. Puleo, and E. Vezzoni, IEEE Transactions on Microwave Theory and Techniques 30(10), 1497-1503 (1982).

[10] TIA/EIA FOTP-175 - chromatic dispersion measurement of singlemode optical fibers by the differential phase shift method, Standard, Telecommunications Industry Association, Washington, DC, 1992.

[11] R. M. Carter, W. N. MacPherson, P. Jaworski, F. Yu, R. J. Beck, J. D. Shephard, and D. P. Hand, Optical Engineering 55, 55 - 55 - 8 (2016).

[12] M. G. Welch, C.E. de Nobriga, R. A. Correa, W. J. Wadsworth, and J. C. Knight, Opt. Express 17(11), 90069011 (2009).

[13] E. Myslivets, N. Alic, and S. Radic, IEEE Photonics Technology Letters 20(21), 1793-1795 (2008).

[14] I. Demirtzioglou, C. Lacava, K. R. H. Bottrill, D. J. Thomson, G. T. Reed, D. J. Richardson, and P. Petropoulos, Opt. Express 26(2), 790-796 (2018).

[15] W. Bogaerts, P. De Heyn, T. Van Vaerenbergh, K. De Vos, S. Kumar Selvaraja, T. Claes, P. Dumon, P. Bienstman, D. Van Thourhout, and R. Baets, Laser \& Photonics Reviews 6(1), 47-73 (2011).

[16] A. Boskovic, S. V. Chernikov, J. R. Taylor, L. GrunerNielsen, and O. A. Levring, Opt. Lett. 21(24), 1966-1968 (1996).

[17] K. Krakenes and K. Blotekjaer, Journal of Lightwave Technology 13(4), 682-686 (1995).

[18] G. D. VanWiggeren and D. M. Baney, IEEE Photonics Technology Letters 15(9), 1267-1269 (2003).

[19] D. B. Neumann and H. W. Rose, Appl. Opt. 6(6), 10971104 (1967).

[20] N. Cooper, J. Woods, J. Bateman, A. Dunning, and T. Freegarde, Appl. Opt. 52(23), 5713-5717 (2013).

[21] H. Cho, R. Arai, H. Suzuki, and M. Takemoto, Journal of The Japanese Society for Non-Destructive Inspection 53(11), 709-714 (2004).

[22] A. Oriana, J. Réhault, F. Preda, D. Polli, and G. Cerullo, J. Opt. Soc. Am. A 33(7), 1415-1420 (2016).
[23] C. Vinegoni, M. Wegmuller, and N. Gisin, IEEE Photonics Technology Letters 13(12), 1337-1339 (2001). 